\title{
New radio sources and the composite structure of component $B$ in the very young protostellar system IRAS 16293-2422
}

\author{
Laurent Loinard \\ Centro de Radiostronomía y Astrofísica, Universidad Nacional Autónoma de México, \\ Apartado Postal 72-3 (Xangari), 58089 Morelia, Michoacán, México; \\ l.loinard@astrosmo.unam.mx \\ Claire J. Chandler \\ National Radio Astronomy Observatory, P.O. Box O, Socorro, NM 87801, USA; \\ cchandle@nrao.edu \\ Luis F. Rodríguez and Paola D'Alessio \\ Centro de Radiostronomía y Astrofísica, Universidad Nacional Autónoma de México, \\ Apartado Postal 72-3 (Xangari), 58089 Morelia, Michoacán, México; \\ l.rodriguez,p.dalessio@astrosmo.unam.mx \\ Crystal L. Brogan \\ National Radio Astronomy Observatory, 520 Edgemont Road, Charlottesville, VA \\ 22903-2475, USA; cbrogan@nrao.edu \\ David J. Wilner and Paul T.P. Ho ${ }^{1}$ \\ Harvard-Smithsonian Center for Astrophysics, 60 Garden Street, Cambridge, MA 02138; \\ dwilner,pho@cfa.harvard.edu
}

\begin{abstract}
In this article, we report high-resolution $\left(\sim 00^{\prime \prime} 1-00^{\prime \prime} 3\right)$, high-sensitivity $(\sim 50-$ $100 \mu \mathrm{Jy}$ beam $^{-1}$ ) Very Large Array 0.7 and $1.3 \mathrm{~cm}$ observations of the young stellar system IRAS 16293-2422 in $\rho$-Ophiuchus. In the $0.7 \mathrm{~cm}$ image, component $\mathrm{A}$ to the south-east of the system looks like its usual binary self. In the new $1.3 \mathrm{~cm}$ image, however, component A2 appears to have split into two subcomponents located roughly symmetrically around the original position of A2.
\end{abstract}

\footnotetext{
${ }^{1}$ Also at: Academia Sinica, Institute of Astronomy and Astrophysics, Taipei 106, Taiwan
} 
This change of morphology is likely the result of a recent bipolar ejection, one of the very first such events observed in a low-mass source. Also in component A, a marginal detection of $0.7 \mathrm{~cm}$ emission associated with the submillimeter component $\mathrm{Ab}$ is reported. If confirmed, this detection would imply that $\mathrm{Ab}$ is a relatively extended dusty structure, where grain coagulation may already have taken place. With an angular size increasing with frequency, and an overall spectra index of 2 , the emission from component $\mathrm{B}$ to the north-west of the system is confirmed to be dominated by optically thick thermal dust emission associated with a fairly massive, nearly face-on, circumstellar disk. In the central region, however, we find evidence for a modest free-free contribution that originates in a structure elongated roughly in the east-west direction. We argue that this free-free component traces the base of the jet driving the large-scale bipolar flow at a position angle of about $110^{\circ}$ that has long been known to be powered by IRAS 16293-2422.

Subject headings: stars: formation - binaries: general - astrometry - radio continuum: stars — stars: individual (IRAS 16293-2422)

\section{Introduction}

A significant fraction of the stars in the Solar neighborhood are known to reside and are suspected to have formed in binary or higher order multiple system, 1 (Duquennoy \& Mayor 1991). Yet, while the formation of isolated Solar-type stars is reasonably well understood (Shu, Adams \& Lizano 1987), our comprehension of the formation of multiple stellar systems remains comparatively limited. Several theoretical mechanisms have been put forward, but observational constraints on the earliest evolutionary phases are scarce owing to the difficulty of identifying and studying the various constituents (disks, jets, etc.) of extremely young systems (for a recent review on the properties of young binaries, see Duchêne et al. 2007). This is because the size scale of, and separation between, these structures is typically of the order of a few to a few tens of astronomical units, and so observations with an angular resolution better than a few tenths of an arcsecond are needed even in the nearest starforming regions. Unfortunately, data with this kind of angular resolution are currently unavailable at the frequencies (mid-infrared, far-infrared, and submillimeter) best matched to the energy output of deeply embedded sources. Such resolutions can only be reached

\footnotetext{
${ }^{1}$ The exact fraction may be smaller than initially anticipated for very low-mass stars (Lada 2006), but it is high for the Solar type objects such as those considered here.
} 
in the optical and near-infrared (but very enshrouded young stars are not detected at these frequencies), and at radio wavelengths $(0.7 \mathrm{~cm} \leq \lambda \leq 6 \mathrm{~cm})$ thanks to the availability of large radio-interferometers. Most of the structures found in young stellar systems do emit in the radio domain, but different mechanisms are at work in different components (thermal dust emission in accretion disks, thermal bremsstrahlung in jets, gyrosynchrotron in magnetically active young stars, etc.). Consequently, a detailed analysis of each radio source found in a given protostellar system has to be carried out before it can be identified -or at least associated- with a specific component.

\section{IRAS 16293-2422}

Located in L1689N, a dark cloud in the $\rho$-Ophiuchus star-forming complex (at $d=$ 120 pc -R.M. Torres et al., in prep), IRAS 16293-2422 is a well-studied very young lowmass protostellar system (e.g. Ceccarelli et al. 2000a). It has been suspected to be multiple since Mundy et al. (1986) and Wootten (1989) showed that it was a double source both at millimeter and centimeter wavelengths. Soon after, it was also found to power a multi-lobe outflow system (Mizuno et al. 1990), comprising two bipolar and one monopolar structures. The two bipolar flows are fairly compact, and at position angles of approximately $60^{\circ}$ and $110^{\circ}$ (Mizuno et al. 1990, Hirano et al. 2001, Castets et al. 2001), whereas the monopolar flow is a parsec-scale, blueshifted feature, located eastward of IRAS 16293-2422 at a position angle of almost exactly $90^{\circ}$ (Mizuno et al. 1990). The lack of a redshifted counterpart toward the west is likely related to the location of IRAS 16293-2422 near the western edge of L1689N. While the two bipolar flows are now generally accepted to be driven by two distinct protostellar sources, it is still debated whether the mono-sided structure is driven by yet another young star in the system, or if it is an extension of the compact outflow at P.A. $110^{\circ}$ (Mizuno et al. 1990, Stark et al. 2004). In the latter situation, the jet powering the east-west flows must either have undergone significant precession, or have been strongly deflected by a condensation of dense gas along its path.

At the best resolution available at centimeter wavelengths, IRAS 16293-2422 is resolved into three radio components (Wootten 1989, Loinard 2002, Chandler et al. 2005). Components A1 and A2 -to the south-east of the system; see Fig. 1- are separated from each other by about 0.34 , and from component $\mathrm{B}$, to the north-west, by about $5^{\prime \prime}$. Component B appears to be well resolved in high-resolution $0.7 \mathrm{~cm}$ observations (Rodríguez et al. 2005), has a spectral index of 2-2.5 across the millimeter and centimeter ranges, and has been interpreted as a nearly face-on, optically thick accretion disk (Rodríguez et al. 2005, Chandler et al. 2005). It has often been associated with the outflow at P.A. $\sim 110^{\circ}$ but significant 
doubts remain about this issue (Chandler et al. 2005).

Although it has been known for a long time to be at the origin of the outflow at P.A. $60^{\circ}$ (Mundy et al. 1986, 1992), the exact nature of the A1/A2 pair remains unclear. Wootten (1989) argued that A2 is the protostellar source driving the outflow and that A1 is a shock feature along that flow. This conclusion was based -in part- on the near-exact alignment with the flow of the segment joining A1 to A2 at the epoch (1986-1987) of the observations published by Wootten (1989). In that scheme, A1 could either be a knot of ionized gas ejected by A2 some time in the past, or the result of the impact of the jet powered by A2 onto dense circumstellar material. In the former case, one would expect A1 to move steadily away from A2 along the direction of the flow, whereas in the latter, no significant motions are expected between A1 and A2. Multi-epoch radio observations with sufficient angular resolution (better than about 0 .'2) to resolve A1 and A2, however, revealed neither of these behaviors (Loinard 2002, Chandler et al. 2005). Instead, they showed that the separation between A1 and A2 has remained constant at about 0..34 in the last 15 years, whereas the position angle between them has changed from less than $50^{\circ}$ in the late 1980 s to about $85^{\circ}$ in 2003 (Loinard 2002, Chandler et al. 2005). This definitely rules out the possibility that A1 is an ejecta from A2, because -as mentioned earlier- one would then have expected constant position angle and increasing separation rather than the opposite. The possibility that A1 is the result of the interaction between a jet powered by A2 and circumstellar material could still be retained if that jet precessed strongly between the late 1980s and 2003, and were now oriented almost exactly east-west (at P.A. $\sim 85^{\circ}$ ). Recent observations, however, have shown that the $0.7 \mathrm{~cm}$ radio emission from A2 (that partly traces free-free radiation from the inner part of the jet) is elongated at P.A. $\sim 60^{\circ}$, very similar to the orientation of the large-scale molecular flow driven from within component A. Thus, it appears that A2 does drive the large-scale flow at P.A. $\sim 60^{\circ}$, and that A1 is not a shock feature along that flow.

The simplest interpretation of the relative motion between A1 and A2 (constant separation and linearly increasing position angle) is that they are two protostellar sources in Keplerian orbit (Loinard 2002). In this hypothesis, the total mass of the system can be estimated from Kepler's third law. Assuming a face-on circular orbit, the mass of the system implied by the motions is $2.7(\mathrm{~d} / 120 \mathrm{pc})^{3} \mathrm{M}_{\odot}$ (where $d$ is the distance -Chandler et al. 2005). For an elliptical orbit, a somewhat smaller value would be permitted, but the total mass has to be larger than $1.4(\mathrm{~d} / 120 \mathrm{pc})^{3} \mathrm{M}_{\odot}$ for the system to remain bound. Moreover, this minimum value is only attained for an extremely eccentric orbit observed exactly at periastron. For less special circumstances, a mass larger than 2 to $3(\mathrm{~d} / 120 \mathrm{pc})^{3} \mathrm{M}_{\odot}$ is required. Finally, an analysis of the absolute proper motions (Loinard 2002, Chandler et al. 2005) shows that, in this orbital motion scenario, the center of mass would have to be very close to A2, so that A2 would have to be significantly more massive than A1. The A1/A2 pair would 
then be a binary system made of a relatively low-mass protostar $\left(M \lesssim 0.5 \mathrm{M}_{\odot}\right)$ in orbit around a somewhat more massive one $\left(M \gtrsim 2 \mathrm{M}_{\odot}\right)$. That binary system would, in turn, be orbited by the protostar associated with component B, and IRAS 16293-2422 would be a hierarchical triple system. Note that if A1 and A2 indeed form a binary system, then the position angle between them should keep increasing monotonically with time. Furthermore, if observations with a quality similar to those used by Loinard (2002) and Chandler et al. (2005) are obtained regularly in the coming decade, it will become possible to fit a Keplerian orbit to the data, and determine accurately the mass of both components.

An alternative to this orbital motion scenario was recently formulated by Chandler et al. (2005). In that proposal, A1 is interpreted as a shock resulting from the impact of a strongly precessing (or wobbling) jet driven by a third -as-yet undetected-protostar in the system. Of course, in this alternative scheme, the change of position angle with time should decelerate, and eventually reverse its course because the jet must oscillate around an equilibrium value. Note that the required fast rate of precession or wobbling (about $40^{\circ}$ in less than twenty years) has never been observed before, and would require the new protostar to be a member of a very tight binary. Given the overall morphology of the system, the most likely situation would be for this new member to be a close companion of A2 -with a separation smaller than $22 \mathrm{AU}(0$ ' 18 if $\mathrm{d}=120 \mathrm{pc}$-Chandler et al. 2005). It is interesting to point out that, in that alternative scheme, there would also be two protostellar sources in the A component and, including component B, IRAS 16293-2422 would again be a hierarchical triple system. Thus, the two scenarios put forth so far call for the existence of three protostellar sources in IRAS 16293-2422. Given that three outflow systems are known to be powered from within IRAS 16293-2422 (see above), it is, of course, tempting to associate each one of the sources with one of the flow features. So far, however, the only secure association is that between A2 and the flow at P.A. $\sim 60^{\circ}$.

In recent high-resolution, submillimeter observations (Chandler et al. 2005), IRAS 162932422 was also found to be a triple source (Aa, Ab, and B -see Fig. 1), but the exact correspondence between the radio and submillimeter sources in the A component is not immediately obvious2. Component Aa is located just about halfway between A1 and A2 (Fig. 1) and it is unclear if it is another protostar in the system, or a blend of roughly equal amounts of emission associated with A1 and A2. If it was a new source, its position roughly 0 "' 2 from A2 would, of course, make it a potential candidate for the companion of A2 driving the precessing jet that impacts circumstellar material at A1. Component Ab, on the other hand,

\footnotetext{
${ }^{2}$ The situation is clear for component B, where the position of the submillimeter and radio sources are almost identical, and the submillimeter flux is in excellent agreement with that expected from the extrapolation of the centimeter data.
} 
is located about 0.'6 to the north-east of component $\mathrm{A}$, and is not detected at any other wavelengths. Since the mechanism at work is almost certainly dust thermal emission, Ab must be a dense dusty structure, and Chandler et al. (2005) argued that it may be associated with a fourth protostar in the system. The absence of centimeter emission, however, shows that it is not associated with a strong ionized wind. Moreover, for thermal dust emission, the spectral energy distribution is expected to be a power law with an index $\alpha$ between 2 and 4. From the $0.5 \mathrm{Jy}$ flux detected at $305 \mathrm{GHz}$, one would expect a $0.7 \mathrm{~cm}$ flux of about 10 mJy if the spectral index is 2 , and $0.2 \mathrm{mJy}$ if $\alpha$ is 4 . A $10 \mathrm{mJy}$ source would have been easily detected in the $0.7 \mathrm{~cm}$ observations reported by Rodríguez et al. (2005) unless the emission were fairly extended $\left(\gtrsim 0.5^{\prime \prime}\right)$ and significantly filtered out. Only a very marginal detection $(2 \sigma)$ would have been obtained in the second case $(\alpha=4)$, but the source would have been detected above $5 \sigma$ for any spectral index smaller than 3.5 (again, unless the emission were extended). Thus, the non-detection of $\mathrm{Ab}$ in the $0.7 \mathrm{~cm}$ data reported by Rodríguez et al. (2005) implies either that the emission is fairly extended, or that the spectral index is larger than 3.5, and the dust consequently largely unprocessed (or a combination of both). Together with the lack of free-free emission, these characteristics argue in favor of either an extremely young protostar, or even a starless condensation.

IRAS 16293-2422 has long been known to exhibit a very active chemistry (e.g. van Dishoeck et al. 1995, Cazaux et al. 2003), and is one of the sources where the hot corinos (Ceccarelli et al. 2007) that have been argued to trace the earliest stages of low-mass starformation have first been identified. The earliest observations (e.g. Ceccarelli et al. 2000b and references therein) did not resolve the various components of IRAS 16293-2422, but several recent high-resolution interferometric millimeter observations (Kuan et al. 2004, Bottinelli et al. 2004, Chandler et al. 2005) have demonstrated that the organic and highly deuterated molecules considered signposts of hot corinos exist in both component A and component B. It appears, therefore, likely that each component harbors at least one very young protostar.

In summary, although it has been extensively studied and several characteristics of IRAS 16293-2422 are now well-established, many other questions remain open about the very nature of several of the sources in the system, and their exact relation to the large-scale outflows in the region. It is important to address these issues, because IRAS 16293-2422 is a rare example of a very young multiple system, that offers a unique opportunity to study the earliest stages of the formation of multiple systems. 


\section{Observations}

In this article, we will present and analyze two new, high-resolution, high-sensitivity, radio continuum observations of IRAS 16293-2422 obtained at 0.7 and $1.3 \mathrm{~cm}$ (Tab. 1). The data were collected with the Very Large Array (VLA) of the National Radio Astronomy Observatory (NRAO ${ }_{3}^{3}$ ) in the most extended $(A)$ and second most extended (B) configurations of the array at $1.3 \mathrm{~cm}$ and $0.7 \mathrm{~cm}$, respectively. The standard VLA continuum frequency setups were used: two frequencies $(22.4851$ and $22.4351 \mathrm{GHz}$ at $1.3 \mathrm{~cm}$, and 43.3149 and $43.3649 \mathrm{GHz}$ at $0.7 \mathrm{~cm}$ ) were observed simultaneously, and in both circular polarizations, with $50 \mathrm{MHz}$ of bandwidth each. The absolute flux density was set using observations of $3 \mathrm{C}$ 286. For improved flux accuracy, we did not assume 3C 286 to be a point source, but instead used a model image provided by NRAO. The phase calibrator was PKS J1625-2527 whose absolute position is expected to be accurate to about 2 milli-arcseconds. The fast-switching technique -that consists of rapidly alternating observations of the source and the phase calibrator with a cycle time of 2 minutes- was used for both observations; this ensured optimal imaging fidelity. The $0.7 \mathrm{~cm}$ data were restored with natural weighting of the visibilities, whereas the $1.3 \mathrm{~cm}$ data were imaged with a robust weighting scheme intermediate between uniform and natural (see Tab. 1 for the corresponding synthesized beams).

To complement these new data, we will also make use of observations previously published in the literature. In particular, we will use the recent $0.7 \mathrm{~cm}$ observations obtained in 2003.47 and reported by Rodríguez et al. (2005), and the $3.6 \mathrm{~cm}$ high-resolution observations obtained in 2003.65, and reported by Chandler et al. (2005). For these two datasets, new images were made from the calibrated visibilities. The $0.7 \mathrm{~cm}$ data from 2003.47 were initially imaged with natural weighting of the visibilities. For the study of component A (which is faint at $0.7 \mathrm{~cm}$ ) as well as for accurate comparison with the new $1.3 \mathrm{~cm}$ data, this image was also smoothed to the resolution of the $1.3 \mathrm{~cm}$ image (Tab. 1) The $3.6 \mathrm{~cm}$ data were imaged with a robust weighting scheme intermediate between natural and uniform.

Our new B-array $0.7 \mathrm{~cm}$ observations were found to produce fluxes systematically $30 \%$ lower than the older A-array observations. This was originally identified by comparing the total flux of component B (which has never been found to be variable in any previous observations) in the two datasets. A similar difference was found when comparing the total flux corresponding to similar baselines and hour angles in our A- and B-configuration observations. This effect clearly cannot be due to missing flux related extended emission, since this would have produced more flux in the B-configuration observations than in the A-array data,

\footnotetext{
${ }^{3} \mathrm{NRAO}$ is a facility of the National Science Foundation operated under cooperative agreement by Associated Universities, Inc.
} 
rather than the observed opposite. Instead, the flux deficit is likely related to the relatively poorer weather conditions during our B-array observations. To account for this effect, the new B-configuration $0.7 \mathrm{~cm}$ data were multiplied by 1.3 before imaging. The only instance when this will be important will be in Sect. 4.3, when we estimate the flux of the possible $0.7 \mathrm{~cm}$ counterpart of Ab.

\section{Results and discussion}

\subsection{Component A}

In our new $0.7 \mathrm{~cm}$ observation (Fig. 2b), component A looks like its usual binary self, with sub-components A1 and A2 clearly resolved. In the new $1.3 \mathrm{~cm}$ image, however, it has clearly become triple (Fig. 2a, particularly the inset). This is the first time that component A appears triple rather than double in a radio image. While the easternmost source is clearly A1, the relation between the two western sources and A2 is a priori quite unclear. Since they are identified here for the first time, we have very little information on the spectral energy distributions of the two western sources. The only statement that can safely be made on their spectral properties is that they are both continuum (rather than spectral) features because they are detected with identical fluxes at both observed frequencies (22.4851 and $22.4351 \mathrm{GHz}$-see Sect. 2). It is also noteworthy that the total flux of component A in our new $1.3 \mathrm{~cm}$ image $(5.2 \pm 0.3 \mathrm{mJy})$ is comparable to the $1.3 \mathrm{~cm}$ flux of component A in all previous observations (see Fig. 7, left panel in Chandler et al. 2005). Thus, while the morphology of A2 has undergone significant changes, its flux has not. This suggests, in particular, that the current overall spectral index of component $\mathrm{A}$ is similar to that found in previous observations. In the centimeter regime, that spectral index is about 0.53 (see Fig. 2, left panel in Chandler et al. 2005) suggesting that free-free is the dominant emission process.

To examine further the relation between the three sources associated with component A in our new $1.3 \mathrm{~cm}$ image and the usual sub-components A1 and A2, it is useful to superimpose our new $1.3 \mathrm{~cm}$ map onto one of the previous high resolution radio images of component A. For this purpose, we will use the smoothed version of the A-array $0.7 \mathrm{~cm}$ image (Sect. 3), because it has been obtained relatively shortly before the $1.3 \mathrm{~cm}$ data presented here, and because it provides a good compromise between angular resolution and sensitivity. Since the absolute positions of the various components of IRAS 16293-2422 change with time (Loinard 2002, Chandler et al. 2005), one has to properly register the images before the superposition. The absolute proper motion of A2 is known to be very similar to that of component B (Chandler et al. 2005). From the four recent observations considered in this 
paper, the proper motion of component B can be calculated to be

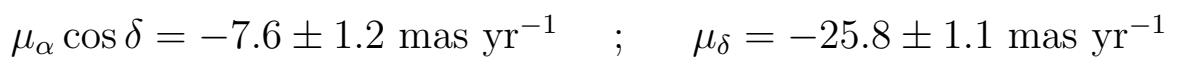

The latter value is somewhat larger than those quoted by Loinard et al. (2002) and Chandler et al. (2005). The reasons for this discrepancy are not entirely clear, but may be related to the fact that the older data used by Loinard et al. (2002) and Chandler et al. (2005) are of lower quality than the more recent observations considered here. In any case, we will use the values quoted above, since they are more appropriate for the comparisons that will be made in this paper between recent observations. Given that the $0.7 \mathrm{~cm}$ image was obtained 2.64 years before the present $1.3 \mathrm{~cm}$ data, it was shifted by -20.1 mas and -68.0 mas in right ascension and declination, respectively, before the superposition. The result of the comparison (Fig. 3) confirms that the easternmost $1.3 \mathrm{~cm}$ component is nearly exactly coincident with the $0.7 \mathrm{~cm}$ source A1, and can indeed be identified with it. Neither of the two western $1.3 \mathrm{~cm}$ sources, however, appears to coincide with source A2. Instead, they are located roughly symmetrically on each side of A2, and we shall refer to them as A2 $\alpha$ and $\mathrm{A} 2 \beta$ in the rest of the paper (Fig. 2). The line joining A2 $\alpha, \mathrm{A} 2$, and A2 $\beta$ (thick dotted line on Fig. 3) is at a position angle of about $62^{\circ}$, remarkably similar to the direction of the large-scale flow known to be driven by A2. This strongly suggests that A2 $\alpha$ and A2 $\beta$ are the result of a recent bipolar ejection by the protostar within A2, the position of which is likely near the cross in Fig. 3. While bipolar ejections are not uncommon in young stellar objects (e.g. Martí et al. 1995, Curiel et al. 2006), they rarely lead to such dramatic morphological changes. In particular, in our case, it seems that very little emission is left at the very position of the protostar, the emission concentrating instead in the two bipolar lobes. This usually does not happen in bipolar ejections by protostars. Also, while bipolar ejections have been observed from relatively massive young stellar system, this is -to our knowledge- the first time that it is seen in such a low-mass source. It is interesting to note that a situation almost exactly opposite of that reported here was recently found in a radio source in Orion. Source "n", which had been bipolar in all previous VLA observations, was indeed found to be single in the 2007 VLA image to be published by L. Gómez et al. (in prep).

A $2 \alpha$ and A2 $\beta$ were clearly not present in previous radio images so if they are indeed ejecta, they must be very recent ones. Water masers associated with component A are found to expand at a velocity projected on the plane of the sky of $40-65 \mathrm{~km}^{-1}$ (Wootten et al. 1999). If this value can be taken as representative of the projected velocity of the jet powered by A2, then it would have taken just about a year for A2 $\alpha$ and A2 $\beta$ to reach the present position. This is consistent with their absence from any previous observation. Note, also, that if $\mathrm{A} 2 \alpha$ and $\mathrm{A} 2 \beta$ are ejecta moving at about $50 \mathrm{~km} \mathrm{~s}^{-1}$ away from the protostar 
associated with A2, then their proper motions should be easily detectable. An observation of IRAS 16293-2422 in the upcoming A configuration of the VLA (around 2007.8), should show $\mathrm{A} 2 \alpha$ and $\mathrm{A} 2 \beta$ displaced from their current position by about 0 .'1.

\subsection{Relative motion between $\mathrm{A} 1$ and A2}

The relative motion between A1 and A2 in the last 15 years or so has been investigated in detail by Loinard (2002) and Chandler et al. (2005). As mentioned earlier, these studies have led to the conclusion that the separation between A1 and A2 has remained constant at about 0 "' 34 , whereas their relative position angle has changed by about $40^{\circ}$ between the late 1980s and 2003. The new $0.7 \mathrm{~cm}$ observation reported here can be used to further monitor the evolution of the relative position of A1 and A2. The value of the position angle found in that observation ( $82 \pm 3^{\circ}$-Fig. 4 ) is similar to those reported for the 2003 observations by Chandler et al. (2005). Obtaining information from the new $1.3 \mathrm{~cm}$ observation is somewhat more difficult because of the change of structure undergone by component A2. In Fig. 4, we report the separation and position angle between $\mathrm{A} 1$ and both $\mathrm{A} 2 \alpha$ and $\mathrm{A} 2 \beta$. As discussed earlier, however, the protostar in A2 is unlikely to be coincident with either of these two sources. Instead, it must be near the cross in Fig. 3, a location roughly equivalent to the mean of the positions of A $2 \alpha$ and A2 $\beta$. The red circles in Fig. 4 indicate the separation and position angle between A1 and the cross in Fig. 3. Taken together, these new observations suggest that the separation between A1 and the protostar associated with A2 has remained constant around $0^{\prime \prime} 34$ in the last few years, whereas their relative position angle is between 80 and $90^{\circ}$. Unfortunately, this does not shed much new light on the relative motion between A1 and A2. In particular it is insufficient to discriminate between the orbital path scenario (where one would expect the position angle to keep increasing linearly) and the precessing/wobbling scheme (where the change of position angle should decelerate and eventually reverse its course). More observations in the coming decade will be needed to settle this issue.

\section{3. $\quad 0.7 \mathrm{~cm}$ emission from component $\mathrm{Ab}$ ?}

We mentioned in Sect. 1 that the submillimeter source Ab, located 0".6 to the northeast of Component A has, to date, never been detected at other wavelengths. To further examine this issue, we searched both of our new images for a counterpart of Aa. While there is clearly no detection in the present $1.3 \mathrm{~cm}$ data, a possible source is detected at the $4 \sigma$ level in our new B-configuration $0.7 \mathrm{~cm}$ dataset (Fig. 5). The best 2-dimensional

Gaussian fit to this structure yields a peak flux density of about $0.76 \mathrm{mJy}^{\mathrm{beam}}{ }^{-1}$, and an 
integrated flux of $1.8 \pm 0.6 \mathrm{mJy}$. Note that the noise level in the $0.7 \mathrm{~cm} \mathrm{~A}$ array observations published by Rodríguez et al. (2005) was $0.1 \mathrm{mJy}^{\text {beam }}{ }^{-1}$, so this source should have been detected in that dataset also if it had been present. The fact that it was not, implies either that the present marginal detection is not real, or that the $0.7 \mathrm{~cm}$ emission associated with $\mathrm{Ab}$ is extended, and was more heavily filtered out in the A array observations published by Rodríguez et al. (2005) than in the present B array data. Deep, C-configuration VLA observations at $0.7 \mathrm{~cm}$ ought to settle this issue. If the marginal detection reported here is real, then the spectral index of the emission associated with component Ab must be smaller than about 3 independently of the extent of the emission. Thus, component Ab would be a relatively extended structure containing somewhat processed dust. Combined with the lack of centimeter emission tracing winds, this would favor a scenario where Ab is a starless condensation rather than a protostar.

\subsection{Component B: disk and jet combined}

Component B is clearly resolved in our new $1.3 \mathrm{~cm}$ observations (Fig. 6.c), and its morphology is very similar to that in the A-configuration $0.7 \mathrm{~cm}$ image smoothed to a similar resolution (Fig. 6.a). It is slightly more compact, however, at $1.3 \mathrm{~cm}\left(0{ }^{\prime \prime} 19 \times 0\right.$ "'15) than at $0.7 \mathrm{~cm}\left(00^{\prime \prime} 21 \times 00^{\prime \prime} 20\right)$. This is consistent with the trend noticed by Chandler et al. (2005) between archival $1.3 \mathrm{~cm}$ and $3.6 \mathrm{~cm}$ data -in the $3.6 \mathrm{~cm}$ image shown in Fig. 1, the source is found to have a deconvolved size of 0 " $16 \times 0$ "' 10 - and suggests that the (linear) angular size of the emission increases roughly as $\nu^{+0.3}$. This increase of the angular size with frequency is opposite to what is expected from optically thick free-free emission. Combined with the measured spectral index in the centimeter regime of about 2 (Chandler et al. 2005), this strongly suggests that the dominant radiation mechanism is optically thick thermal dust emission as proposed before (Rodríguez et al. 2005, Chandler et al. 2005). Clearly, to make the emission optically thick, the circumstellar disk associated with component B must be quite dense and massive (Chandler et al. 2005). Since the emission is well-resolved in our data, we can investigate if and how the spectral index depends on radius. We find that the spectral index in the very inner region (at $R<0$ ". 1 ) is $1.4 \pm 0.2$, whereas it is $2 \pm 0.2$ in the region 0 "' $1<\mathrm{R}<0$ ". 2 (Fig. 6.d). The spectral index outside of $\mathrm{R}=0$ "' 2 becomes difficult to calculate reliably because the emission fades quickly, but it is clearly larger than 2. The lower value of the spectral index near the center of component B suggests that the optically thick thermal dust emission in these regions coexists with a lower spectral index component. The most likely candidate for this additional component would be free-free radiation from the base of an ionized wind. Such a wind would indeed be expected to exist in an actively-accreting protostar like that believed to reside at the center of component B. 
It is interesting in this context, to compare the structure of the inner and outer regions of component B. The image most suitable for that purpose is the full-resolution $0.7 \mathrm{~cm}$ image obtained in the A configuration of the VLA (Fig. 6.b), because it provides the best compromise between sensitivity and angular resolution. It is noteworthy in that image that the outer isophotes are elongated roughly in the north-south direction (at a position angle marginally positive), whereas the inner isophotes are elongated roughly in the east-west direction (at P.A. $\sim 110^{\circ}$ ) very similar to that of one of the large-scale outflows known to originate from within IRAS 16293-2422. This would be consistent with the idea that component $\mathrm{B}$ is at the origin of that flow; the radio source associated with it being the superposition of a compact thermal jet at P.A. $\sim 110^{\circ}$, and a somewhat extended disk roughly perpendicular to the jet. It is puzzling in that scenario, however, that no highvelocity emission is detected towards component B in any molecular transition (Chandler et al. 2005). If a thermal jet is associated with the protostar near the center of component $\mathrm{B}$, one would indeed expect to find entrained high velocity gas associated with it. Future, high-resolution, millimeter and submillimeter spectral observations might help resolve this puzzle.

\section{Conclusions and perspectives}

The new observations presented in this paper provide a number of interesting clues on the nature and properties of the various radio sources in IRAS 16293-2422.

1. Component A2 is definitely confirmed to be at the origin of the compact bipolar flow at P.A. $\sim 60^{\circ}$. Indeed, in the new $1.3 \mathrm{~cm}$ image, A2 has undergone a dramatic morphological change, being now composed of two emission peaks distributed roughly symmetrically around A2 at a position angle of almost exactly $60^{\circ}$. We argue that this change reflects a recent bipolar ejection, an interpretation that will be confirmed if forthcoming observations reveal the appropriate proper motions.

2. Unfortunately, the new 0.7 and $1.3 \mathrm{~cm}$ data do not allow us to characterize much better the relative motion between $\mathrm{A} 1$ and $\mathrm{A} 2$. It remains unclear, therefore, whether the changes in relative position observed in the last two decades reflect an orbital motion, or the precession or wobbling of a jet. It will be important to settle that issue to decide where the third star in the system is, and if that star might be at the origin of the third outflow structure known to exist in IRAS 16293-2422. Further observations in the next ten years or so ought to provide a definite answer.

3. A marginal detection of $0.7 \mathrm{~cm}$ emission near the position of the submillimeter source 
$\mathrm{Ab}$ is reported. It would be very important to further characterize the very nature of this source, and its relation to the other members of the system. If the present marginal detection is real, it should be easily confirmed by future deep $0.7 \mathrm{~cm}$ observations in the $\mathrm{C}$ configuration of the VLA.

4. The radio emission from component $B$ at the northwest of the system is confirmed to be dominated by optically thick thermal dust emission, suggesting that the radio source in that component traces an optically thick accretion disk. At the very center of that structure, however, the lower observed value of the spectral index suggests the existence of a modest contribution from a thermal jet. The orientation of the inner $0.7 \mathrm{~cm}$ isophotes further suggests that this free-free component traces the base of the compact bipolar flow at P.A. $\sim 110^{\circ}$. This association would appear reasonable since the flow at P.A. $\sim 110^{\circ}$ has a dynamical age of only a few thousand years (Mizuno et al. 1990), and component B is known to harbor organic and deuterated molecular species expected to be present only in the youngest protostellar sources (Cazaux et al. 2003, Kuan et al. 2004, Bottinelli et al. 2004, Chandler et al. 2005).

L.L., L.F.R., and P.A. acknowledge the financial support of DGAPA, UNAM and CONACyT, México. D.J.W. acknowledges partial support from NASA Origins of Solar Systems Program Grant NAG5-11777.

\section{REFERENCES}

Bottinelli, S., et al. 2004, ApJ, 617, L69

Castets, A., Ceccarelli, C., Loinard, L., Caux, E., \& Lefloch, B. 2001, A\&A, 375, 40

Cazaux, S., Tielens, A. G. G. M., Ceccarelli, C., Castets, A., Wakelam, V., Caux, E., Parise, B., \& Teyssier, D. 2003, ApJ, 593, L51

Ceccarelli, C., Caselli, P., Herbst, E., Tielens, A. G. G. M., \& Caux, E. 2007, Protostars and Planets V, 47

Ceccarelli, C., Castets, A., Caux, E., Hollenbach, D., Loinard, L., Molinari, S., \& Tielens, A.G.G.M. 2000, A\&A, 355, 1129

Ceccarelli, C., Loinard, L., Castets, A., Tielens, A. G. G. M., \& Caux, E. 2000, A\&A, 357, L9 
Chandler, C.J., Brogan, C.L., Shirley, Y.L., \& Loinard, L. 2005, ApJ, 532, 371

Curiel, S., et al. 2006, ApJ, 638, 878

Duchêne, G., Delgado-Donate, E., Haisch, K.E., Jr., Loinard, L.; Rodríguez, L. F. 2007, Protostars \& Planets V, B. Reipurth, D. Jewitt, and K. Keil (eds.), University of Arizona Press, Tucson, 279

Duquennoy, A., \& Mayor, M. 1991, A\&A, 248, 485

Hirano, N., Mikami, H., Umemoto, T., Yamamoto, S., \& Taniguchi, T. 2001, ApJ, 547, 899

Kuan, Y.-J., et al. 2004, ApJ, 616, L27

Lada, C.J. 2006, ApJ, 640, L63

Loinard, L. 2002, RMAA, 38, 61

Marti, J., Rodriguez, L. F., \& Reipurth, B. 1995, ApJ, 449, 184

Mizuno, A., Fukui, Y., Iwata, T., Nozawa, S., \& Takano, T. 1990, ApJ, 356, 184

Mundy, L. G., Wilking, B. A., \& Myers, S. T. 1986, ApJ, 311, L75

Mundy, L. G., Wootten, H. A., Wilking, B. A., Blake, G. A., \& Sargent, A. I. 1992, ApJ, 385,306

Rodríguez, L.F., Loinard, L., D’Alessio, P., Wilner, D.J., \& Ho, P.T.P. 2005, ApJ, 621, L133

Shu, F.H., Adams, F.C., \& Lizano, S. 1987, ARAA, 25, 23

Stark, R., et al. 2004, ApJ, 608, 341

van Dishoeck, E. F., Blake, G. A., Jansen, D. J., \& Groesbeck, T. D. 1995, ApJ, 447, 760

Wootten, A. 1989, ApJ, 337, 858 
Table 1: Observing logs

\begin{tabular}{lrrrllll}
\hline \hline Date & Project & $\begin{array}{c}\nu \\
(\mathrm{GHz})\end{array}$ & Conf. & $\begin{array}{l}\text { Weighting/ } \\
\text { Smoothing }\end{array}$ & Synthesized beam & Figs & Refs $^{\mathrm{a}}$ \\
\hline 2003.47 & AL592 & 43.2299 & A & Nat. & $0^{\prime \prime} 09 \times 0 . \prime 05 ;+1.8^{\circ}$ & $6 \mathrm{~b}$ & 1,2 \\
2003.47 & AL592 & 43.2299 & A & Nat./Smo. & $0^{\prime \prime} 13 \times 0 \times 06 ;-0.5^{\circ}$ & $3,6 \mathrm{a}$ & 3 \\
2003.65 & AL589 & 8.4601 & A & Rob. & $0^{\prime \prime} 39 \times 0 . \prime 19 ;+6.8^{\circ}$ & 1 & 2 \\
2005.20 & AC778 & 43.2299 & B & Nat. & $0^{\prime \prime} 30 \times 0 \times 17 ;-1.9^{\circ}$ & $2 \mathrm{~b}, 5$ & 3 \\
2006.11 & AL672 & 22.4601 & A & Rob. & $0^{\prime \prime} 13 \times 0 . \prime 06 ;-0.5^{\circ}$ & $2 \mathrm{a}, 3,6 \mathrm{c}$ & 3 \\
\hline \hline
\end{tabular}

${ }^{a} 1=$ Rodríguez et al. (2005); $2=$ Chandler et al. (2005); 3=This work 


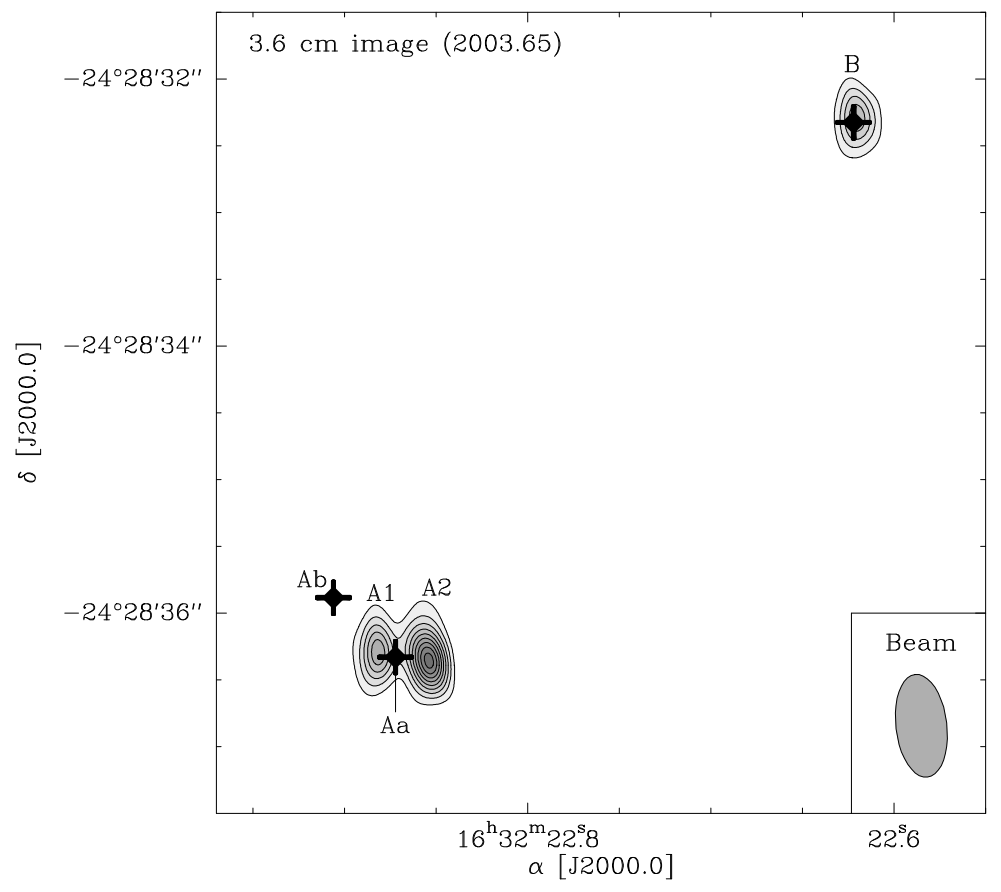

Fig. 1.- 3.6 cm VLA A-array image of IRAS 16293-2422 obtained in 2003.65 (Chandler et al. 2005) and restored with a weighting scheme intermediate between natural and uniform. The first contour and the contour interval are $0.1 \mathrm{mJy}^{\text {beam }}{ }^{-1}$, while the noise level is $0.02 \mathrm{mJy}$ beam $^{-1}$. The synthesized beam, shown at the bottom-right corner, is 0 "' $39 \times$ 0 . $19 ;+6.8^{\circ}$. The radio components $\mathrm{A} 1, \mathrm{~A} 2$, and $\mathrm{B}$ are labeled, and the position of the submillimeter sources Aa, Ab, and B are indicated. The errors on the submillimeter source positions are significantly smaller than the crosses indicating their locations. 

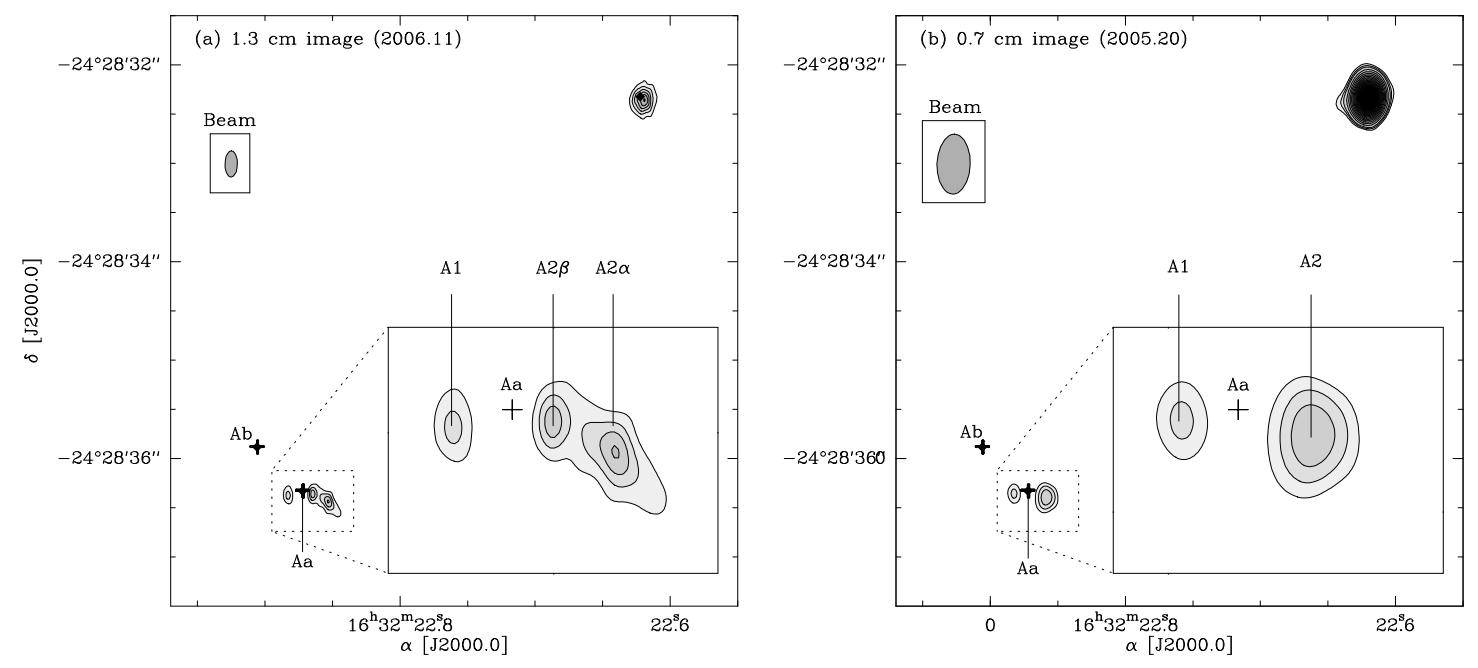

Fig. 2.- (a) $1.3 \mathrm{~cm}$ continuum observation of IRAS 16293-2422 obtained in 2006.11 restored with a weighting scheme of the visibilities intermediate between natural and uniform. The first contour is at $0.2 \mathrm{mJy} \mathrm{beam}^{-1}$ and the contour step is $0.3 \mathrm{mJy}^{\mathrm{beam}}{ }^{-1}$, while the noise is $0.04 \mathrm{mJy}_{\text {beam }^{-1}}$. The synthesized beam, shown near the top-left corner, is $0.13 \times 0$ ". 06 ; $-0.5^{\circ}$. (b) $0.7 \mathrm{~cm}$ image of IRAS $16293-2422$ obtained in 2005.20 in the B configuration of the VLA when restored with natural weighting. The first contour is at $1.5 \mathrm{mJy} \mathrm{beam}^{-1}$ and the contour step is $0.3 \mathrm{mJy}_{\text {beam }}{ }^{-1}$, while the noise is $0.18 \mathrm{mJy} \mathrm{beam}^{-1}$. The synthesized beam, shown near the top-left corner, is $0^{\prime \prime} 30 \times 0$. . $17 ;-1.9^{\circ}$. In both panels, the positions of the submillimeter sources $\mathrm{Aa}$ and $\mathrm{Ab}$ are indicated, and insets provide zooms on component A. Note that component A is clearly triple in the $1.3 \mathrm{~cm}$ image, while it remained double in the $0.7 \mathrm{~cm}$ map. 


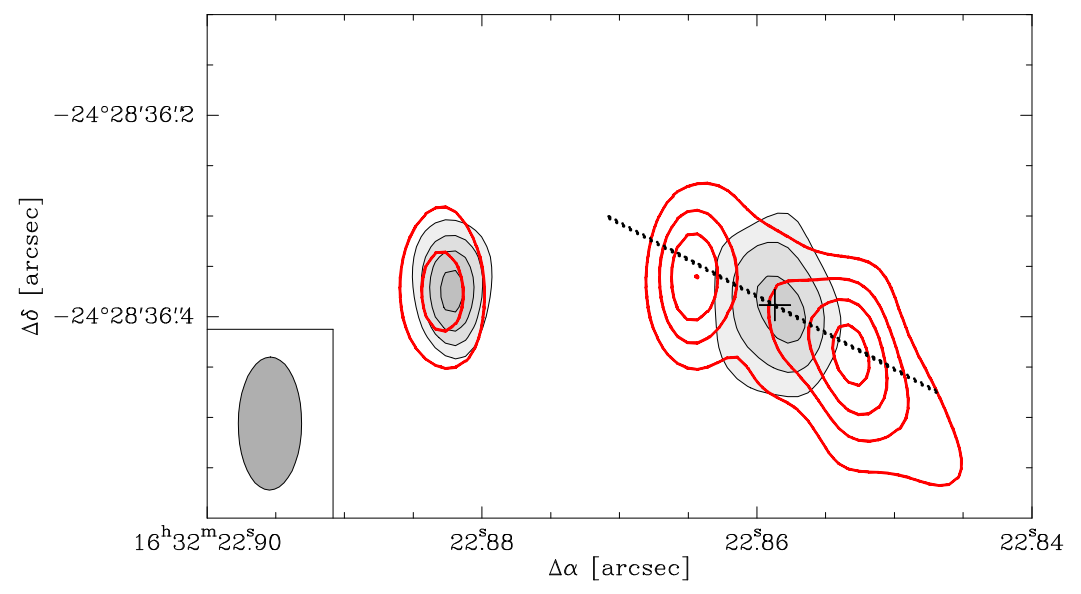

Fig. 3.- Comparison between the smoothed $0.7 \mathrm{~cm}$ image of component A obtained in 2003 (black contours and grey scale) and our new $1.3 \mathrm{~cm}$ image (red contours). The angular resolution of the two images is identical $\left(0.13 \times 0.0^{\prime \prime} 06 ;-0.5^{\circ}\right)$ and is shown at the bottomleft. Translational shifts have been applied to account for the overall proper motion of the region. The first contour and the contour interval for the $1.3 \mathrm{~cm}$ image are the same as in Fig. 2; they are at 0.6 and $0.15 \mathrm{mJy}^{\text {beam }}{ }^{-1}$, respectively, for the $0.7 \mathrm{~cm}$ image. The dotted line is at a position angle of $62^{\circ}$ 

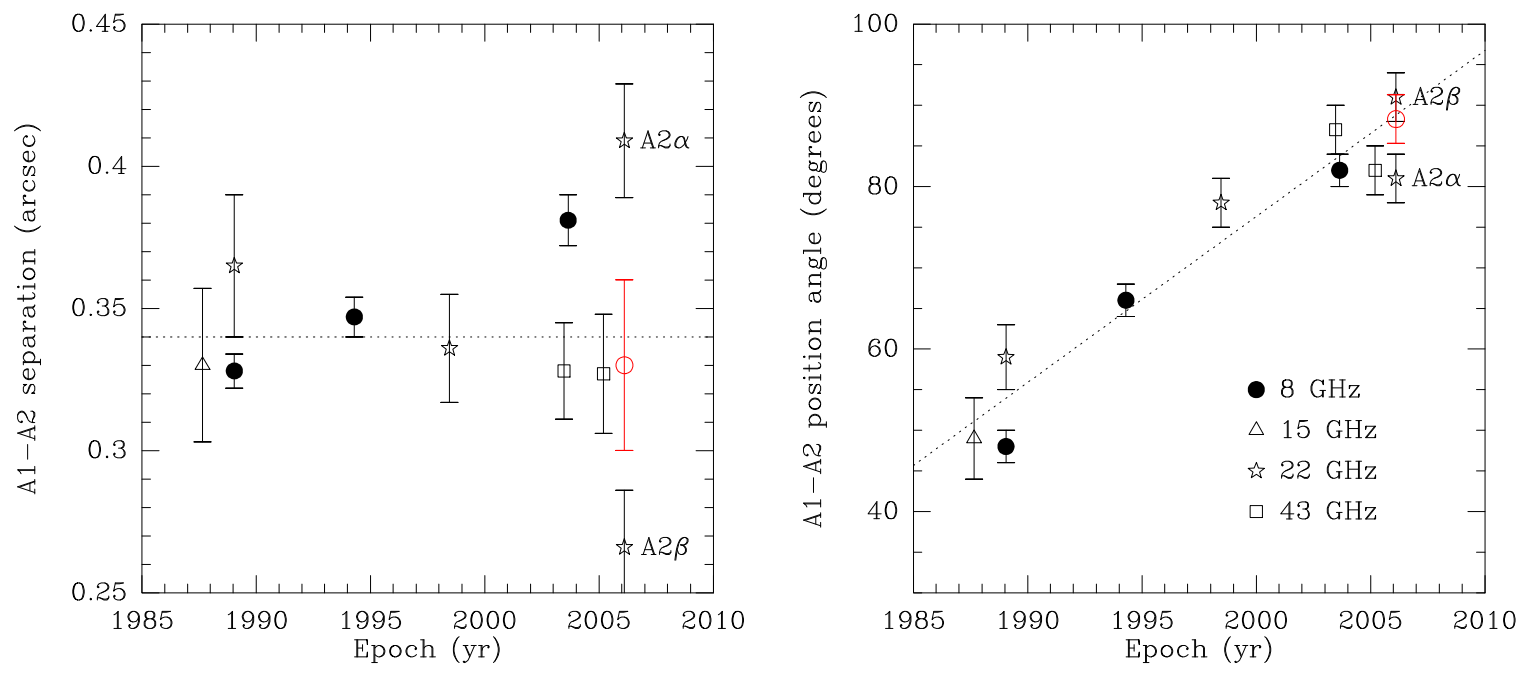

Fig. 4.- Evolution of the separation (left) and relative position angle (right) of the A1/A2 pair. For the $1.3 \mathrm{~cm}$ data obtained in 2006.11, three points are shown: in black the parameters for A2 $\alpha$ and A2 $\beta$, and in red those corresponding to the position were the protostar is inferred to be. The dotted line in the left panel shows a constant separation of 0. "34, whereas the dotted line in the right panel shows the best linear fit to all the data points but those corresponding to the 2006.11 image. 


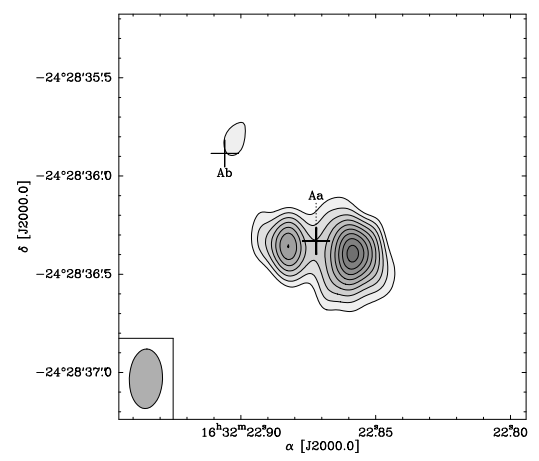

Fig. 5.- $0.7 \mathrm{~cm}$ image of component A. The first contour is at $0.7 \mathrm{mJy}_{\mathrm{beam}}{ }^{-1}$, and the contour interval is $0.2 \mathrm{mJy}_{\text {beam }}^{-1}$, while the noise is $0.18 \mathrm{mJy}_{\text {beam }}{ }^{-1}$. The synthesized beam, shown at the bottom-left corner is $0 . \prime 30 \times 0 . \prime 17 ;-1.9^{\circ}$. Note the existence of a positive signal near the position of Ab. 

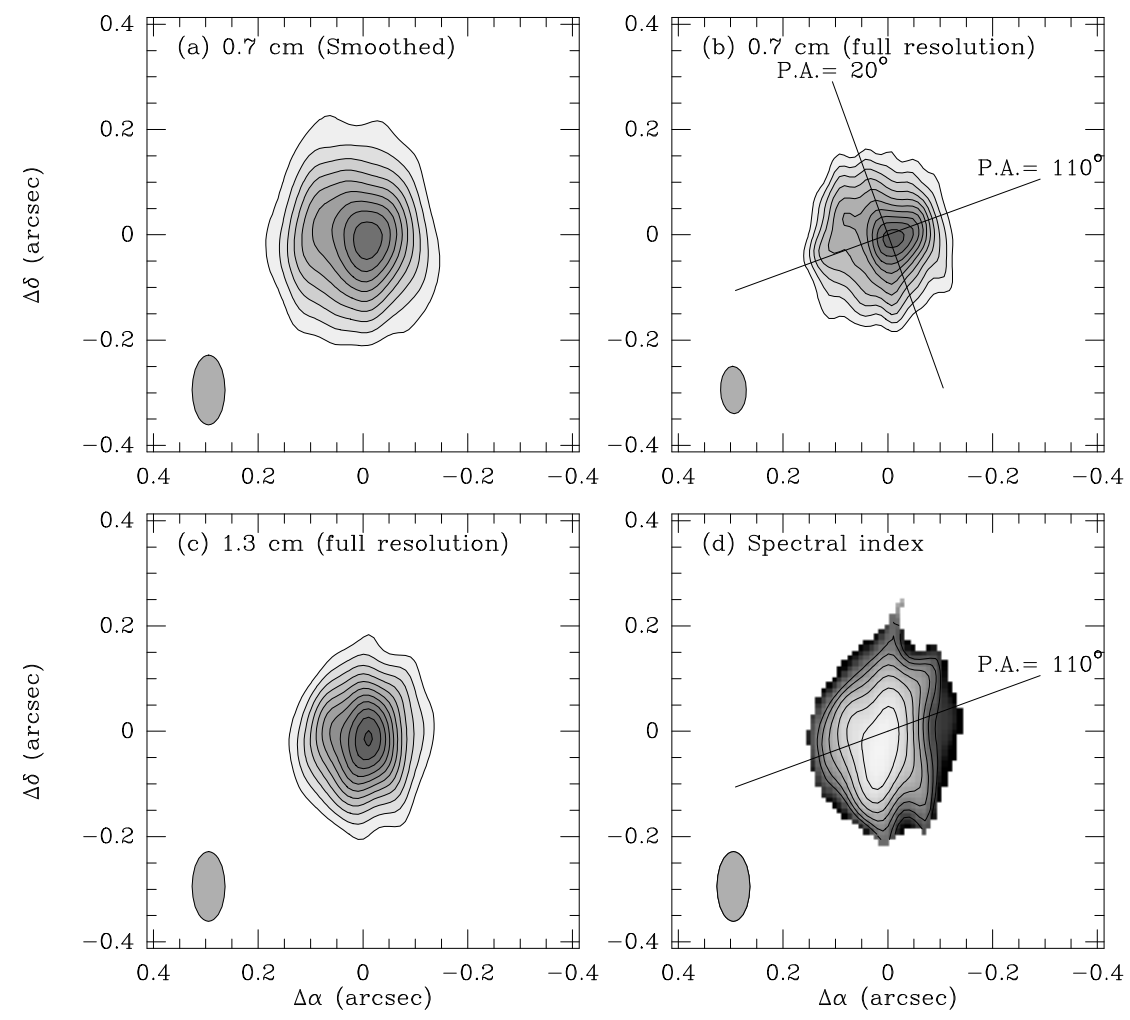

Fig. 6.- Comparison between high angular resolution images of component B in IRAS 16293-2422 obtained at $0.7 \mathrm{~cm}$ (panels a-b) and $1.3 \mathrm{~cm}$ (panel c). In panel (b), we show the full-resolution $0.7 \mathrm{~cm}$ combined $\mathrm{A}+\mathrm{B}$ configuration image. The first contour is at $0.6 \mathrm{mJy}_{\text {beam }}{ }^{-1}$ and the contour interval is $0.2 \mathrm{mJy}_{\text {beam }}^{-1}$, while the noise level is $0.12 \mathrm{mJy}_{\text {beam }}{ }^{-1}$. The synthesized beam, shown at the bottom-right corner is 0 " $09 \times 0$ " 05 ; $+1.8^{\circ}$. In panel (c) we show the full resolution $1.3 \mathrm{~cm} \mathrm{~A}$ configuration image. The first contour is at $0.2 \mathrm{mJy}_{\text {beam }}{ }^{-1}$ and the contour interval is $0.15 \mathrm{mJy}_{\text {beam }}^{-1}$. In panel (a) we show the $0.7 \mathrm{~cm}$ image smoothed to the resolution of the $1.3 \mathrm{~cm}$ image. The first contour 